\title{
Suppression of Pulse Interference in Partial Discharge Measurement Based on Phase Correlation and Waveform Characteristics
}

\author{
Shengyou Gao, Qi Wang, Junfeng Gui, Yunfeng Xia, Jianhua Lin, Jianhua Huang and Xiyang Chen \\ Electrical Engineering Department, Tsinghua University, Beijing 100084, China
}

Received: November 18, 2014 / Accepted: January 05, 2015 / Published: March 31, 2015.

\begin{abstract}
Partial discharge measurement is one of the most effective methods to find insulation defects and early failure of high voltage power equipments. The accuracy is significantly reduced by the interference in the partial discharge on-site detection or on-line monitoring, especially by the pulse interference. This paper studies the phase correlation of some types of typical partial discharge pulses and their characteristics in time domain and frequency domain. By collecting enough partial discharge pulse data, the correlation coefficient can be calculated based on both phase correlation and waveform similarity. The type of pulse will be determined by the scope of the calculated correlation coefficient. The pulses with very strong correlation will be identified as periodic pulse interference. The pulses with very weak correlation will be identified as random pulse interference. Only the pulses whose correlation coefficients fall into a specific range will be identified as partial discharge signals. In laboratory, simulated pulse interference is injected into measurement circuit, and typical partial discharge pulses are sampled by a high-speed acquisition system. The pulse interference can be effectively separated from partial discharge signals by correlation coefficient.
\end{abstract}

Key words: Correlation coefficient, partial discharge, phase correlation, pulse interference, waveform characteristic.

\section{Introduction}

Some defects within high voltage electrical apparatus will cause partial discharge at high electric field [1]. Under certain conditions, partial discharge could lead to insulation deterioration, even breakdown. Partial discharge onsite detection or on-line monitoring could find early defects or potential fault of the operating equipments. This is very important to ensure the reliability of power systems. In the environment with strong electromagnetic interference, a large amount of interference may be mixed into the partial discharge signals, which will influence the result of the test. These disturbances can be roughly divided into three categories: periodic interference, continuous pulse interference and white noise [2].

Corresponding author: Shengyou Gao, senior engineer, research fields: on-line monitoring and diagnosis for power equipment. E-mail: eeagsy@mail.tsinghua.edu.cn.
Continuous periodic interference mainly comes from communication systems which have a fixed frequency and bandwidth. Filtering or wavelet transform methods are usually used to suppress them [3-5]. White noise commonly exists in electronic circuit. It has a constant power spectral density and wide spectrum. It is difficult to achieve the desired results by using the traditional frequency domain method. Therefore, the method of wavelet analysis with consideration of both time domain and frequency domain characteristics has been widely adopted [6-9].

Pulse interference is very similar to partial discharge signals. In time domain, it has a short duration. In frequency domain, it has a very wide frequency range [10]. Pulse disturbance that periodically appears in a certain phase is called periodic pulse interference, such as thyristor rectifier, periodically breaking switch etc. The phases of some pulse interference are random, such as electric welding machine, electric spark discharge. For 
this type of interference, some time-domain methods are usually adopted [11-13]. For example, differential equilibrium, polarity identification, time-domain windowing, etc. But the-results are not always ideal. The reason is that the pulse interference, especially the random pulse interference, has very similar characteristics to partial signals in time domain. With the development of high-speed acquisition technology, the interference suppression mean based pulse waveform characteristics is gradually applied [14-17].

In this paper, the phase correlation of partial charge pulses caused by typical defects within XLPE (cross-linked polyethylene) cable is studied. At the same time, the differences between time domain and frequency domain characteristic among some types of partial discharges and interference pulses are compared. Five hundreds continuous power cycles of partial discharge data are acquired and analyzed based on the phase correlation and the waveform characteristics.

For a certain pulse, all the cycles except the one where it is located will be scanned, in order to find the pulses with both phase correlation and waveform similarity. In each cycle, at most one pulse will be used. Therefore, the total number of pulses that meet the requirements is within 0 and 499 . Generally, the total number is normalized by the cycle number, and the result is called correlation coefficient. Only the pulses whose correlation coefficients fall into a certain scope will be-identified as partial discharge signals.

\section{Phase Correlation of Partial Discharge Pulse}

It is well known that, internal and surface discharge signals appear mostly within the $0^{\circ}-90^{\circ}$ or $180^{\circ}-270^{\circ}$ phase. Unless it is in a very serious situation, the discharge will not be extended to other phases. However, the pulses caused by corona discharge usually exist around $90^{\circ}$, and they are almost symmetric to $90^{\circ}$. When the voltage is high enough, a few pulses with large amplitude will appear in the positive half cycle. Fig. 1 shows the pattern of three basic types of discharge: internal, surface and corona. As shown in Fig. 1, regardless of the discharge type, the discharge pulses always appear in a certain range of phase, and a discharge pulse may occur again near the same phase in a few frequency cycles as follows. For example, the discharges in 500 cycles which are continuously sampled will be studied. One pulse in first cycle is marked as pulse A. In following 499 cycles, the pulses whose phase difference with pulse A within $\pm 1^{\circ}$ will be found. In each cycle, if more than one such pulses are found, the pulse that has the least phase difference will be used and the others will be ignored. The total number of the found pulses is marked as $\mathrm{N}$. Obviously, the $\mathrm{N}$ falls into [0, 499]. Fig. 2 shows the partial discharge PRPD (phase resolved partial discharge pattern) sampled from a $4 \mathrm{~m}$ long XLPE cable whose insulation is scratched in the outer semi conductive fracture.

Table 1 shows the number of pulses having phase correlation with five randomly selected pulses.

As a comparison, Table 1 also shows the numbers of pulses of SCR (silicon control) interference and random pulse interference which have phase correlation with those five randomly selected pulses, respectively.

As shown in Table 1, although partial discharge pulses have some strong phase correlation, their phases distributions are still somewhat dispersed. On the other

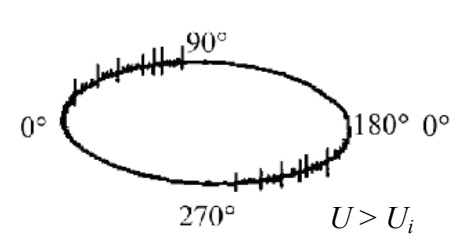

(a)

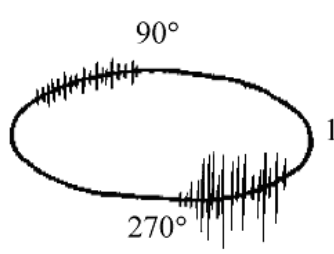

(b)

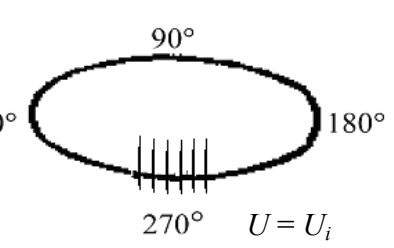

(c)

Fig. 1 Partial discharge pattern of basic types: (a)—internal; (b)—surface; (c)—corona. 


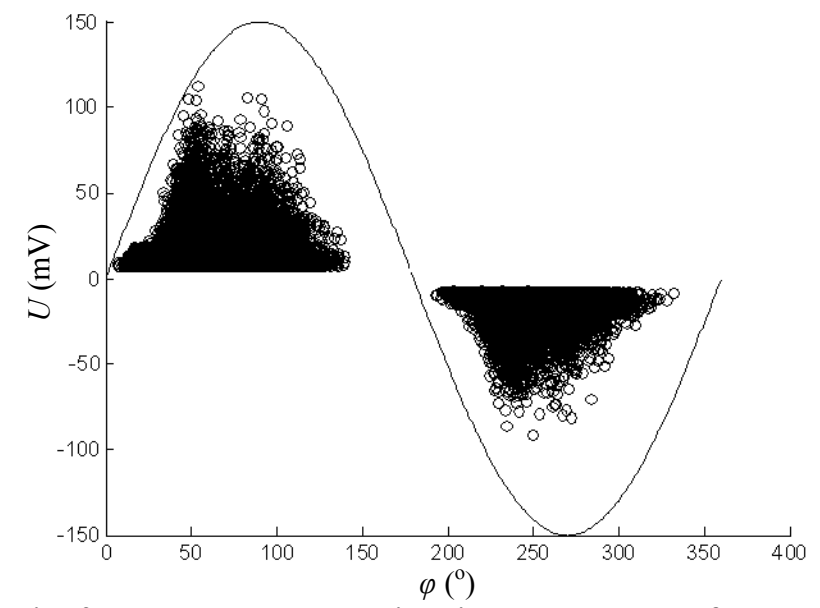

Fig. 2 Phase resolved partial discharge pattern of XLPE cable with defect.

Table 1 Distribution of three types of pulses.

\begin{tabular}{llllll}
\hline & 1 & 2 & 3 & 4 & 5 \\
\hline Partial discharge & 56 & 120 & 86 & 128 & 92 \\
Periodic interference & 499 & 499 & 498 & 499 & 499 \\
Random interference & 5 & 4 & 6 & 4 & 5 \\
\hline
\end{tabular}

hand, the periodic pulse interferences appear in a fixed phase of each cycle and the dispersity is very small. Therefore, the number of those periodic interference pulses with correlation coefficient is greater than the number of partial discharge pulses. Random interference pulses have random phases, so the number of such pulses with correlation coefficient is far less than the above two types of pulses. So, based on the number of pulses with phase correlation coefficient, we can differentiate discharge pulses, periodic interference pulses and random pulses. Of course, the premise is to get enough sample pulses.

\section{Waveform Characteristic of Partial Discharge Pulse}

There are some limitations to solely rely on the phase correlation. The reason is that if the phases of periodic interference pulses overlap with the phase of certain discharge pulses, the accuracy of the test will be significantly affected. Therefore, it is necessary to introduce additional features to identify and separate these pulse interferences from discharges signals.

Researches show that, there are some differences between the pulse waveforms of different types of discharge and interference. Usually, the equivalent time and equivalent frequency of each of the waveform are used to describe the time domain and frequency domain characteristics of the waveform. Equivalent time is defined as Eq. (1):

$$
T=\sqrt{\int_{0}^{T W}\left(t-t_{0}\right)^{2} S_{\mathrm{N}}(t)^{2} \mathrm{~d} t}
$$

where, $S_{\mathrm{N}}(t)$ is the time domain representation through normalization processing, $T W$ is the pulse duration, $t_{0}$ is the time center of the pulse signal, according to Eq. (2) is calculated:

$$
t_{0}=\int_{0}^{T} t\left|S_{\mathrm{N}}(t)\right|^{2} \mathrm{~d} t
$$

Equivalent frequency as defined in Eq. (3) is shown below:

$$
F=\sqrt{\int_{0}^{\infty} f^{2}\left|S_{\mathrm{N}}(f)\right|^{2} \mathrm{~d} f}
$$

where, $S_{\mathrm{N}}(f)$ is the Fourier transform of the time domain expression for the normalized $\mathrm{S}_{\mathrm{N}}(t)$.

The TF (time-frequency) spectra can be plotted based on equivalent time and equivalent frequency. Fig. 3 shows the time domain waveform of three basic types of discharge and the SCR interference, and Fig. 4 is the TF pattern. Further screening can be carried out based on these waveforms characteristics in time domain and frequency domain.

\section{Algorithm Description}

The following is the anti-interference algorithm based on integrated pulse phase and waveform characteristics:

(1) The discharge pulses in $M$ (500, for example) cycles are continuously sampled. The total number of pulses is marked as N. For each pulse, five parameters are recorded: amplitude $A$, phase $\varphi$, equivalent time width $T$, equivalent frequency $F$ and trig time $t$;

(2) For pulse $i(i=1,2, \ldots, N)$, search all the cycles except the one where pulse $i$ is located (total of $M-1$ cycles), find the pulses whose phase difference with pulse $i$ is within $\pm 1^{\circ}$. 


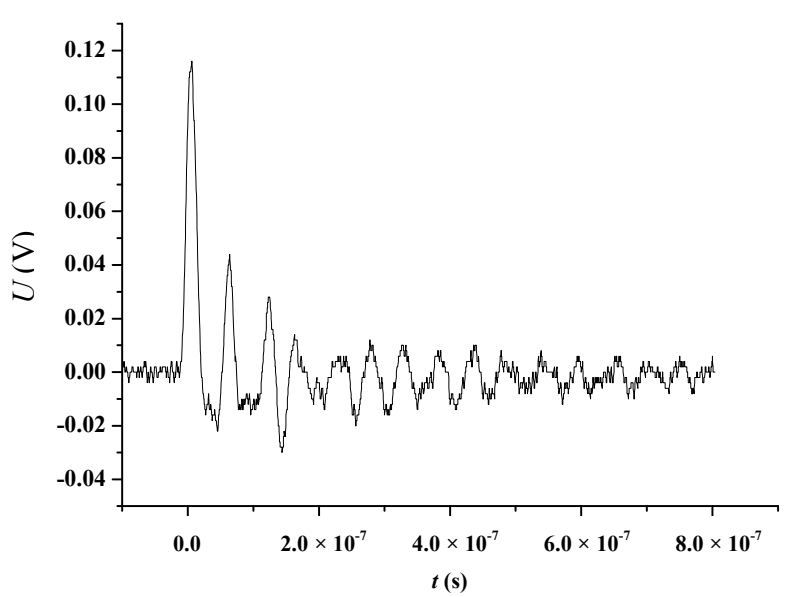

(a)

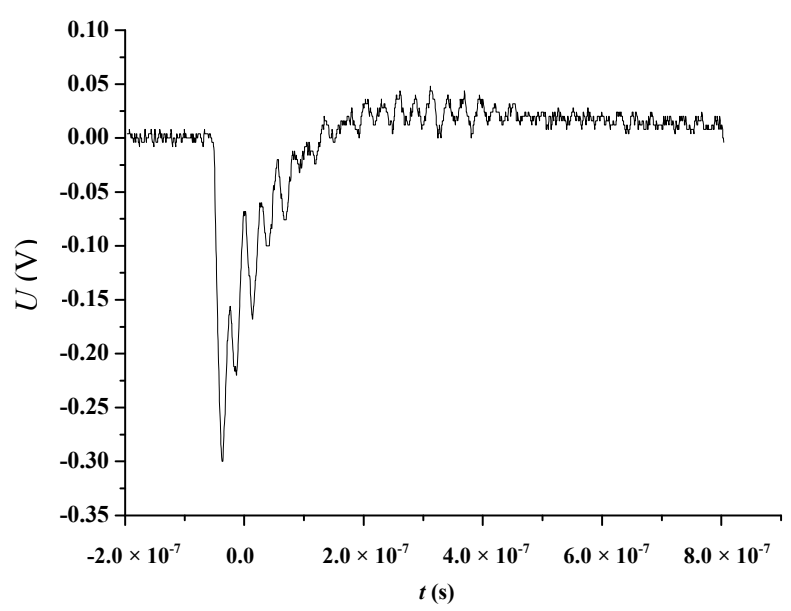

(c)

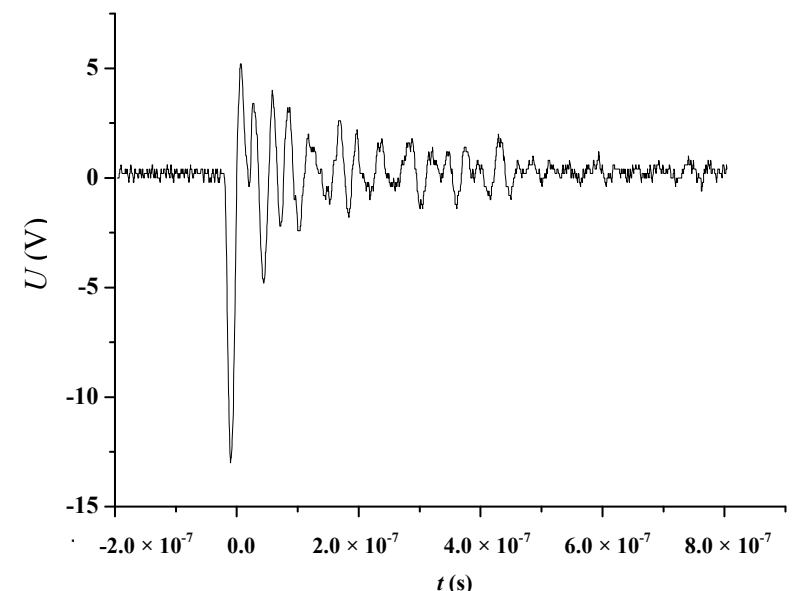

(b)

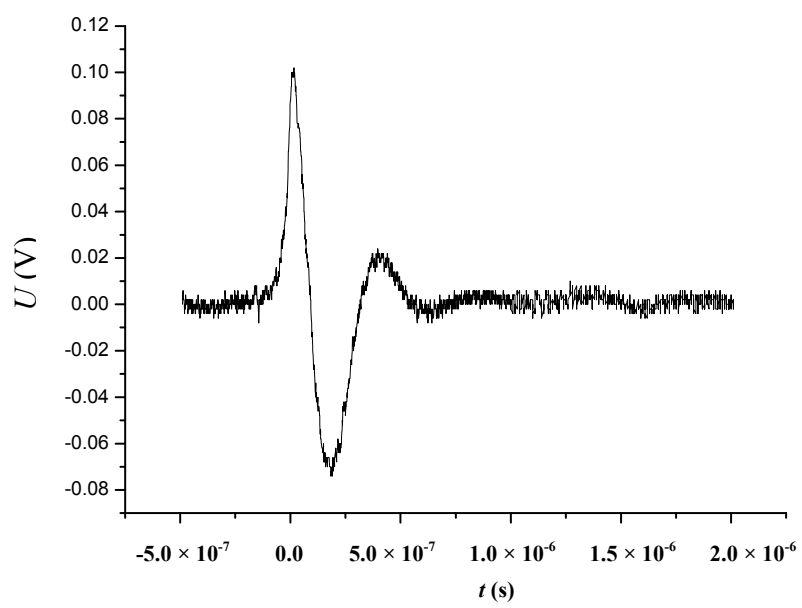

(d)

Fig. 3 Waveform of three basic partial discharge and interference caused by silicon controlled rectifier: (a)—internal; (b)—urface; (c)—corona; (d)—interference.

For each found pulse, the correlation is calculated according to the equivalent time width and equivalent frequency. The distance between object pulse $i$ and pulse $j$ is calculated by Eq. (4):

$$
d=\sqrt{\left(T_{j} / T_{i}-1\right)^{2}+\left(F_{j} / F_{i}-1\right)^{2}}
$$

The $T_{i}$ and $F_{i}$ are the equivalent time width and equivalent frequency of object pulse $i$, respectively. The $T_{j}$ and $F_{j}$ are the equivalent time width and equivalent frequency of pulse $j$, respectively.

For a given threshold $d_{\theta}$, the pulse $j$ will be considered to have correlation with object pulse if $d<$ $d_{\theta}$. If there are more than one pulses meeting the condition in one cycle, only the pulse with minimum $d$ value will be used. In another word, there is at most one matched pulse in one cycle.

For each cycle, if there is one matched pulse in it, the total number of matched pulses $n_{i}$ will be increased by one. By the above description, the total number $n_{i}$ should meet the constraint of $0 \leq n_{i} \leq M-1$.

The total number of matched pulses is normalized by $M$ and the result is defined as correlation coefficient $r_{i}$. The pulse type could be determined according to the criteria listed in Table 2.

\section{Implementation and Verification}

In the laboratory tests that are carried out on the same cable model with Fig. 2, the vertical resolution of the acquisition system is 12 bit, and the sampling rate is $100 \mathrm{MSa} / \mathrm{s}$. The high-frequency current sensor is set 


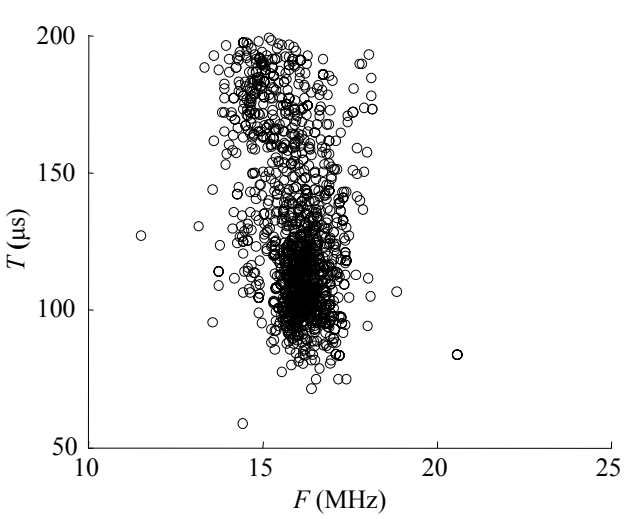

(a)

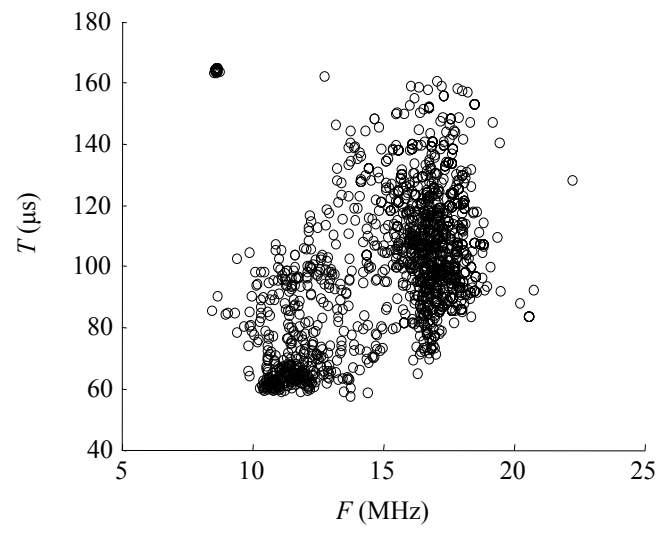

(c)

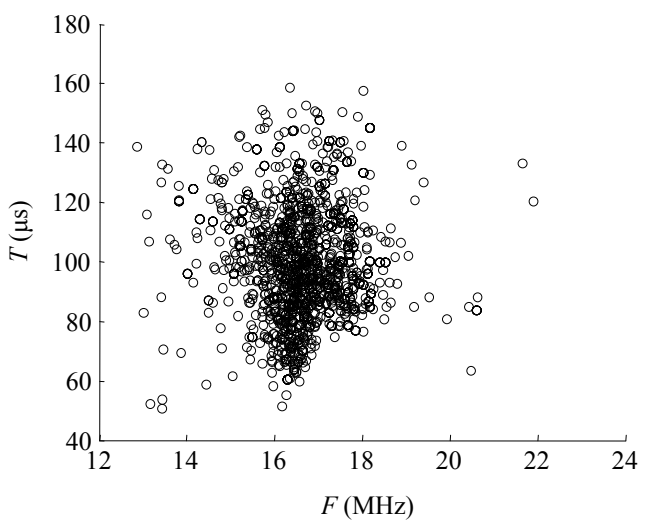

(b)

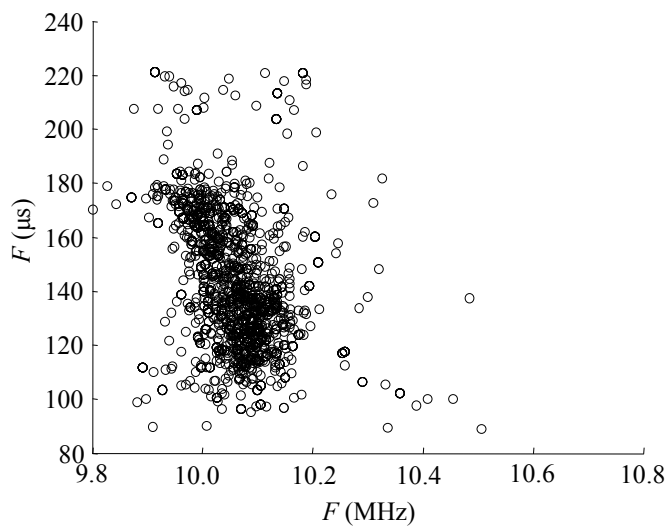

(d)

Fig. 4 TF of three basic partial discharges and interference caused by silicon controlled rectifier: (a)—internal; (b)—surface; (c)_corona; (d)-interference.

Table 2 Pulse type criteria based on correlation coefficient.

\begin{tabular}{llll}
\hline$r$ & $r \geq 0.9$ & $0.1 \leq r<0.9$ & $r<0.1$ \\
\hline Type & Periodic interference & Partial discharge & $\begin{array}{l}\text { Random } \\
\text { interference }\end{array}$ \\
\hline
\end{tabular}

to the grounding line to detect the partial discharge signals.

A power cord with thyristor rectifier power is passed through the high-frequency current sensor for generating periodic pulse interferences. Fig. 5 is a PRPD pattern. The percentages in different correlation coefficient regions calculated based on the above algorithm are shown in Table 3.

When SCR power cord is removed, but other test conditions remain the same, the percentages of pulses of the calculated correlation coefficients are shown in Table 4.

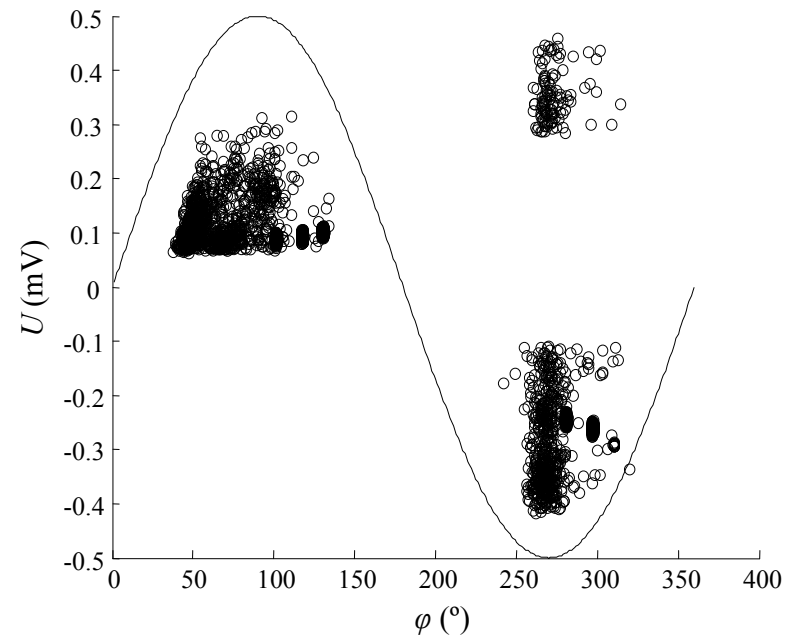

Fig. 5 PRPD with periodic pulse interference.

Table 3 Pulse percentages in each correlation coefficient regions (with periodic interference).

\begin{tabular}{llll}
\hline$r$ & $r \geq 0.9$ & $0.1 \leq r<0.9$ & $r<0.1$ \\
\hline Percentages (\%) & 65.3 & 32.2 & 2.5 \\
\hline
\end{tabular}


Table 4 Pulse percentages in each correlation coefficient regions (without periodic interference).

\begin{tabular}{llll}
\hline$r$ & $r \geq 0.9$ & $0.1 \leq r<0.9$ & $r<0.1$ \\
\hline Percentages (\%) & 0 & 92.9 & 7.1 \\
\hline
\end{tabular}

As can be seen from the test results, most of the pulses whose correlation coefficient are greater than 0.9 , are the periodic interference caused by thyristor rectifier, therefore they can be removed.

A pulse with the width of $2 \mu$ s and the repetition frequency of $51 \mathrm{~Hz}$ is output from the signal generator. It is passed through the high frequency current sensor after being transformed into current. The reason of selecting $51 \mathrm{~Hz}$ repetition rate is to ensure that the phase of the output pulse frequency is not fixed. Therefore it can simulate random phase pulses to some extent. Fig. 6 is PRPD spectra obtained. The pulse percentages in different correlation coefficient regions are shown in Table 5 .

When the generator is turned off, but other test conditions remain the same, the percentages of pulses of the calculated correlation coefficients are shown in Table 6.

As shown in Table 6, random noises have relatively small correlation coefficients, so they can be removed.

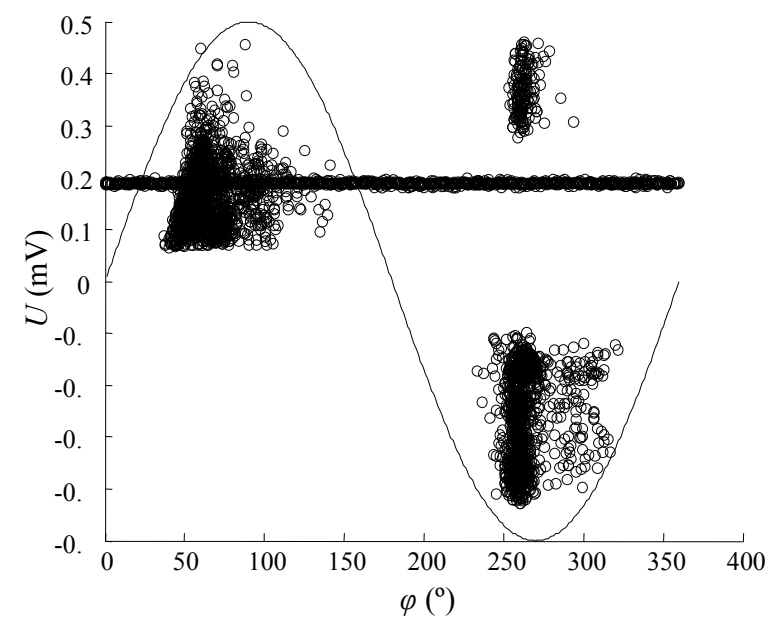

Fig. 6 PRPD with random pulse interference.

Table 5 Pulse percentages in each correlation coefficient regions (with random interference).

\begin{tabular}{llll}
\hline$r$ & $r \geq 0.9$ & $0.1 \leq r<0.9$ & $r<0.1$ \\
\hline Percentages (\%) & 0 & 54 & 46 \\
\hline
\end{tabular}

Table 6 Pulse percentages in each correlation coefficient regions (without random interference)

\begin{tabular}{llll}
\hline$r$ & $r \geq 0.9$ & $0.1 \leq r<0.9$ & $r<0.1$ \\
\hline Percentages (\%) & 0 & 92 & 8 \\
\hline
\end{tabular}

\section{Conclusions}

(1) Theoretical analysis and simulation experiments show that partial discharge pulses, periodic pulse interference and random pulse interference have some differences in terms of phase correlation;

(2) Different types of partial discharge and interference pulses have different waveform characteristics, which can be used to exclude several types of pulses;

(3) By only using the phase correlation method, it is hard to identify the interference pulses that have an overlapping phase with those partial discharge pulses. On the other hand, by only using time domain and frequency domain characteristics, it is hard to separate interference pulses from those partial discharge pulses that have similar waveform characteristics. The combination of these two methods can overcome the shortcomings stated above. As a result, we can greatly improve the accuracy of identifying interference signals.

\section{References}

[1] Changrong, Q., and Naiqing, W. 1994. Electrical Equipment and Testing Technology of Partial Discharge. Beijing: China Machine Press.

[2] Changchang, W., Zhongdong, W., and Fuqi, L. 1995. "Anti-interference Techniques Used for On-line Partial Discharge Monitoring." Journal of Tsinghua University (Science and Technology) 35 (4): 69-74.

[3] Chengjun, H., and Weiyong, Y. 2003. "Study of Adaptive Filter Algorithm Based on Wavelet Analysis in Suppressing PD's Periodic Narrow Bandwidth Noise." In Proceedings of the CSEE (Chinese Society for Electrical Engineering), 23 (1): 107-11.

[4] Nagesh, V., and Gururaj, B. 1993. "Evaluation of Digital Filters for Rejecting Discrete Spectral Interference in On-site PD Measurements." IEEE Transactions on Electrical Insulation 28 (1): 73-85.

[5] Koepf, U., and Feser, K. 1995. "Rejection of Narrow-Band Noise and Repetitive Pulses in On-site PD Measurements." IEEE Transactions on Dielectrics and 
Electrical Insulation 2 (3): 433-47.

[6] Ma, X., Zhou, C., and Kemp, I. J. 2002. "Automated Wavelet Selection and Thresholding for PD Detection." IEEE Transactions on Dielectrics and Electrical Insulation 18 (2): 37-45.

[7] Wu, Z., and Huang, N. E. 2004. "A Study of the Characteristics of White Noise Using the Empirical Mode Decomposition Method." Proceedings of the Royal Society A 460 (2046): 1597-611.

[8] Xiaoxing, Z., Junjie, Z., and Nan, L. 2011. "Block Thresholding Spatial Combined De-noising Method for Suppress White-Noise Interference in PD Signals." High Voltage Engineering 37 (5): 1142-8.

[9] Werle, P., Akbari, A., and Borsi, H. 2002. "Enhanced Online PD Evaluation on Power Transformers Using Wavelet Techniques and Frequency Reject Ion Filter for Noise Suppression." In Proceedings of the 2002 IEEE International Symposium on Electrical Insulation, 195-8.

[10] Stephan, H., Hans, G. K., and Wolfgang, K. 2005. "Advanced Suppression of Stochastic Pulse Shaped Partial Discharge Disturbances." IEEE Transactions on Dielectrics and Electrical Insulation 12 (2): 265-75.

[11] Yanqun, L., Yang, X., and Jiangjing, C. 2010. "Study on Anti-interference Technique of On-line Partial Discharge
Detection for XLPE Power Cable Joints." Guangdong Electric Power 23 (3): 54-59/75.

[12] Gang, L., and Demin, T. 1998. "The Polarity Discrimination Method of Single Point Signal in PD Measurement." High Voltage Engineering 24 (3): 83-6.

[13] Lei, S., Ming, Y., and Jue, W. 2001. "Application of Antijamming Measure of Differential Balanced Method in PD On-line Monitoring of Transformer." Transformer 38 (3): 37-40.

[14] Ling, R., Shengyou, G., and Zhong, Zl. 2009. "Partial Discharge Detection System Based on Very Wide Bandwidth Pulse Current Method." Advanced Technology of Electrical Engineering and Energy 28 (3): 54-7.

[15] Cavallini, A., Montanari, G. C., and Contin, A. 2003. "A New Approach to the Diagnosis of Solid Insulation Systems Based on PD Signal Inference." IEEE Electrical Insulation Magazine 19 (2): 23-30.

[16] Hongxia, L., Yong, Q., and Linpeng, Y. 2011. "Suppression of Stochastic Pulses Interference in XLPE Cables Based on Fuzzy Clustering." Electrical Automation 33 (2): 76-78/81.

[17] Lin, R., Qijia, X., and Shengyou, G. 2010. "Detection and Identification Technologies of Wide Band Partial Discharge." High Voltage Engineering 36 (10): 2473-7. 